\title{
LA GESTIÓN DE RECURSOS: ¿QUÉ PUEDEN HACER LAS PYMES QUE LAS GRANDES ORGANIZACIONES NO PUEDEN, ANTE LA ADAPTABILIDAD EN UN ESCENARIO DE CRISIS?
}

\author{
Amigo, Adriana \\ Páez, Guillermo Néstor \\ Marchetti, Daniel Franco
}

\section{Resumen:}

Las PyMEs, tienen características singulares en sus procesos estratégicos, sus relaciones más informales que formales; la intuición ante los procesos decisorios, la figura paternalista del dueño frente a las nuevas generaciones, entre otros. Estas variables que marcan su cultura empresarial, quizás les planteen desafíos y oportunidades en estos escenarios de imprevisibilidad e incertidumbre. Nuevos interrogantes a resolver serán el cómo se comportarán ante la adaptabilidad, la flexibilidad la empatía con el cliente, el compromiso social, para moldear una estrategia única que fortalezca la propuesta de valor y le permita asegurar su ventaja competitiva sostenible.

Palabras claves: PyMEs - Procesos - Singularidad - Imprevisibilidad - Desafíos Valor - Ventaja - Competitiva - Sostenible

\section{Abstract:}

SMEs have unique characteristics in their strategic, processes, their relationships more informal than formal; the intuition before the decision-making processes, the paternalist figure of the owner in front of the new generations, among others. These variables that mark their business culture, perhaps pose challenges and opportunities in the scenarios of unpredictability and uncertainty. New questions to be resolved, will be how they will be flexibility, empathy with the client, social commitment, to shape a unique strategy that strengthens the value proposition and allows it to ensure its sustainable competitive advantage

Keywords: SMEs - Processes - Unique - Unpredictability - Challenges - Value Advantage - Competitive - Sustainable 


\section{INTRODUCCIÓN}

\section{LAS OPORTUNIDADES QUE NOS PLANTEAN LOS ESCENARIOS DE CRISIS.}

Adaptarse y cambiar con agilidad es hoy uno de los imperativos estratégicos más importantes de las empresas en el entorno VUCA ${ }^{1}$. Supone un gran desafío en un entorno cambiante donde adquiere relevancia moverse con agilidad y ofrecer nuevas respuestas en el contexto y condiciones actuales del COVID19, pero significa una oportunidad para las PYMES teniendo en cuenta sus características prevalentes: su versatilidad, flexibilidad y agilidad en términos de oportunidad con respuestas rápidas e innovadoras.

Fardelli y Corropolese (2012) al estudiar los procesos de toma de decisiones estratégicas en empresas PyMEs identificaron algunas dimensiones para su análisis partiendo de los disparadores (internos y externos) para la toma de decisiones, la relación interactiva entre subprocesos de generación de información y la deliberación y la generación de alternativas de opciones estratégicas.

Estos tres subprocesos (no lineales) dependen tanto de las características personales del empresario PyME como así también de las capacidades y recursos internos de la firma, en un contexto determinado.

A diferencia de las grandes empresas, en las PyMEs estos procesos se ven condicionados por las reducidas capacidades de absorción ${ }^{2}$, por las dificultades para delegar en la toma de decisiones y por la preminencia del empresario PyME (ownermanager) como aquel que lleva adelante en última instancia la formulación de la estrategia. Fardelli y Corropolese (2012) destacan algunos aspectos en las PyMEs como:

- Perfil y características personales del empresario PyME: Comprende los aspectos de la trayectoria personal, educativa y profesional del empresario. Asimismo, incluye su conocimiento del sector y la experiencia acumulada en gestión de empresas y/u organizaciones empresariales. Tiene en cuenta los componentes de la idiosincrasia del empresario.

- Características del proceso de toma de decisiones estratégicas: Implica el grado de racionalidad y sistematización del proceso de toma de decisiones, las fuentes de información y la generación del conocimiento (relación entre lo tácito-codificado).

\footnotetext{
${ }^{1}$ Entorno VUCA comienza a ser utilizado en los años noventa y es un acrónimo inglés de cuatro términos: Volatilily, Uncertainty, Complexity y Ambiguity

${ }^{2}$ La capacidad de absorción refiere a la habilidad de una empresa para reconocer el valor de información nueva, externa a la empresa, asimilarla y aplicarla. Constituye parte del proceso de aprendizaje, en cuanto refleja su habilidad para identificar, asimilar y explotar conocimiento del entorno, siendo crítica para la supervivencia a largo plazo.
} 
- Comprensión del entorno: Es entendido aquí como la percepción y "recorte" del entorno por parte del empresario y/o la firma al momento de desarrollar el proceso de toma de decisiones estratégicas.

\section{LA GESTIÓN DE RECURSOS Y SU INFLUENCIA EN LA GENERACIÓN DE COMPETENCIAS CENTRALES}

La empresa es un equilibrio entre su frente interno y su frente externo.

En el frente externo, la empresa se enfrenta con sus oportunidades, amenazas, su entorno y su compromiso social.

En el frente interno, la empresa define su negocio, con alta percepción de sus clientes y responde a sus objetivos en función del desarrollo de esas capacidades únicas que permiten convertir a su oferta en una propuesta de valor única.

Según Munuera Alemán y Rodríguez Escudero, (2007), cualquier recurso o capacidad no es fuente de ventaja, su verdadero valor estriba en su sustentabilidad.

La sustentabilidad se logra sobre la base de cuatro características, (Amigo; 2012):

- Recursos valorables

- Recursos escasos

- Recursos difíciles de imitar

- Recursos sin sustitutos

Estas características deben coexistir de manera simultánea, para que la ventaja competitiva que dichas características genera, sean sostenibles en el tiempo y no representen una ventaja competitiva temporal.

Según Hamel y Prahalad, (1999) una empresa debe concebirse no sólo como una cartera de productos sino también como una cartera de competencias. Y si una empresa no tiene en cuenta su cartera de competencias asume el riesgo de perder su propio negocio, a pesar de las oportunidades que se le presenten en el frente interno.

Básicamente una empresa que desconoce el potencial que generan sus propias capacidades asume seis riesgos:

1. Paralizar sus propias oportunidades de crecimiento. Es común delimitar los espacios del mercado, sin tener en cuenta si ese espacio se puede sostener con propias ofertas de valor.

2. Asignar sus competencias a segmentos equivocados, esto significa que por ejemplo la empresa invierte en tecnología en mercados hipercompetitivos, a destiempo y desconociendo un futuro de endeudamiento.

3. Debilitar las competencias a medida que la empresa se fragmenta en unidades de negocios. 
4. Depender de proveedores equivocados, lo que lleva a una cadena de valor equivocada.

5. Si el crecimiento depende en gran medida de la inversión en competencias centrales, el desconocer el grado de cuidado que las mismas requieren compromete la propia misión corporativa.

6. La sorpresa de la aparición de competidores que vislumbraron la ausencia de valor, sostenida en esas competencias no desarrolladas por la empresa.

Luego el proceso de desarrollo de las competencias esenciales, se basa en la identificación, la adquisición, la utilización y la protección de la diferenciación lograda por dichas competencias.

La empresa debe tener muy en claro si esas competencias se desarrollarán para satisfacer las necesidades de un mercado actual o nuevo. También deberá definir si para atender las necesidades de un mercado actual o nuevo, debe desarrollar aún más sus potencialidades actuales o invertir en potencialidades nuevas.

Este proceso puede generar cuatro estrategias de competencias/mercado:

\begin{tabular}{|l|l|l|}
\hline & \multicolumn{1}{|c|}{ Mercado actual } & \multicolumn{1}{c|}{ Mercado Nuevo } \\
\hline Competencia actual & $\begin{array}{l}\text { Rellenar el mercado con } \\
\text { fortalezas actuales }\end{array}$ & Desarrollo \\
\hline Competencia nueva & Desarrollo de producto & Innovación y diversificación \\
\hline
\end{tabular}

El desarrollo de una competencia actual, requiere de:

- Disponibilidad de dicha competencia.

- Que la empresa tenga total dominio de su potencial, para no invertir inútilmente en un recurso que no garantiza el agregado de valor.

- Identificación de las competencias obsoletas, cuya amortización genera un costo hundido sin reconversión.

El desarrollo de una competencia nueva, requiere de:

- Accesibilidad a dicha competencia sin afectar el índice digerible de endeudamiento de la empresa.

- Conocimiento de los requerimientos del mercado para invertir en la competencia justa para agregar la oferta justa al mercado correcto.

- El perfil competitivo y el nivel de crecimiento del mercado, determinará la 
capacidad de reconversión de dicha competencia, en tanto la inversión represente una apuesta al futuro, basado en la posibilidad de inversión y generación de tasa de retorno sustentable.

La empresa debe acompañar este proceso de apalancamiento con la estructura organizativa que sostenga un sistema de información, tal que permita el proceso de control de gestión para la sustentabilidad de la estrategia de crecimiento.

Ahora bien, en este proceso de apalancamiento de competencias, ¿qué pasa con la cultura empresarial? Pues el proceso de desarrollo de una competencia actual ó una nueva competencia, representa un proceso de erogación que impacta en la visión económica, financiera y patrimonial de la empresa; por lo que la asignación de recurso que dicho proceso significa, implica que a veces determinadas áreas o gerencias se vean perjudicadas a favor de otras, con su correspondiente consecuencia cultural y política.

Entonces, en nuestro proyecto, se está investigando si las pequeñas y medianas empresas seleccionadas en la muestra:

1. Tienen un programa de competencias esenciales.

2. Lo están aplicando a políticas de crecimiento.

3. Ese proceso se basa en un apalancamiento genuino.

En consecuencia, si el éxito de la estrategia de la empresa, depende del logro de la ventaja competitiva sostenible, según Munuera Alemán y Rodríguez Escudero, (2007) se deberán examinar sus recursos y habilidades, los que se tornan cada vez, más importantes, cuanto más difíciles de conocer, son para la compete

\section{LAS VENTAJAS $Y$ DESVENTAJAS DE LAS PEQUEÑAS ORGANIZACIONES PARA GESTIONAR RECURSOS}

Una mirada sobre la gestión de los recursos en la PyME, considerando recursos y capacidades como dos niveles de análisis diferentes, es la que ha planteado su relación con la estrategia y su identificación a partir de la distinción entre recursos tangibles e intangibles, mediante el concepto de rutinas organizativas. Así mismo, los problemas que plantean la identificación y medición de los activos intangibles de la empresa o capital intelectual.

La evaluación del carácter estratégico de los recursos y capacidades se puede realizar en función de los criterios que permiten obtener la ventaja competitiva (escasez y relevancia), así como de aquellos que inciden en el mantenimiento de la misma (durabilidad, transferibilidad, imitabilidad, sustituibilidad y complementariedad) y de la 
problemática relativa a la apropiación de las rentas asociadas a dicha ventaja competitiva (apropiabilidad).

En cuanto al recurso intangible humano, la generación interna de recursos y capacidades requiere de una estructura organizativa, una política de recursos humanos y una cultura organizativa que incentive el transmitir y compartir conocimiento entre los integrantes de la PyME, de esa forma se lo puede considerar como una ventaja competitiva.

Prahalad y Hamel (1999) postulan que esta ventaja se observa en las PyMEs cuando analizamos su estructura organizativa interna que es esencialmente flexible, flexibilidad que facilita el flujo de conocimientos y el aprendizaje organizativo, mediante unidades organizativas construidas en torno a las competencias esenciales en las que el principio de interrelación prevalece sobre el de autonomía para lograr un aprendizaje entre pares donde fluye el conocimiento de unas a otras (en conjunción con una coordinación horizontal, la creación de equipos de trabajo interdepartamentales e interdisciplinarios que promuevan la cooperación entre unidades). Esta ventaja se potencia si se articula con una cultura organizacional que sostenga políticas que promocionen valores y creencias para favorecer la incorporación de nuevos conocimientos, motivados por la mejora constante y el aprendizaje permanente.

Otra mirada sobre la gestión de los recursos en las PyMEs, podemos hacerla considerando el modelo de Amigo (2009; 2012; 2017), donde los recursos, en un escenario de crisis, tienen una muy diferente performance según cual analicemos.

En cuanto a los recursos financieros, dada la experiencia de crisis cíclicas en Argentina, las PyMEs no han sido importantes tomadoras de crédito, su nivel de endeudamiento promedio es bajo y, por consiguiente, su capacidad para tomar endeudamiento es importante. La aversión al financiamiento externo, tanto a través de entidades financieras como mediante el mercado de capitales, limitó la posibilidad de inversión en planta y equipo. De cualquier manera, en un nuevo contacto de crisis como el de la pandemia del Covid19, esta situación opera como una ventaja.

Dependiendo de la actividad de las PyMEs, los Recursos Físicos tienen diferentes niveles de importancia. La flexibilidad se manifestó con rapidez en algunas actividades para reutilizar sus recursos físicos para nuevos procesos: varias empresas de la actividad de indumentaria, tanto de ropa como de zapatos, viraron rápidamente sus procesos a la fabricación de tapabocas, barbijos y ropa del personal de la salud. Lo mismo ocurrió con empresas del rubro de refrigeración, que optaron por reconvertirse en suministradores de equipos para la atención hospitalaria. Asimismo, la pandemia aceleró procesos que algunas empresas venían gestando por lo cual, algunas 
actividades que venían modificando procesos o tercerizándolos, lo comenzaron a hacer, o bien, cambiaron sus actividades administrativas y comerciales a home-office. Por el lado de los Recursos Humanos, el entrenamiento y experiencia de los empleados, determinan las habilidades disponibles para la empresa. Ante una crisis se pone en juego la capacidad de adaptabilidad de los empleados y tal como expresa Amigo (2009), este recurso determina la flexibilidad estratégica de la empresa para mantener su ventaja competitiva. La capacitación en herramientas digitales así como la formación en habilidades soft son dos instrumentos que pueden garantizar la sustentabilidad de la empresa en un contexto de crisis.

Por otro lado, los Recursos Tecnológicos, especialmente aquellos relacionados con derechos de propiedad intelectual que la empresa hubiera logrado desarrollar aportan también a la adaptabilidad de la misma. Aquellas organizaciones que venían invirtiendo en recursos para la innovación, tanto en forma privada como a través de modelos de asociación público privada, se encuentran en una mejor posición para enfrentar las crisis.

La propuesta de valor que la empresa haya logrado ser percibida por sus clientes, si es así, seguramente se habrá transformado en una ventaja competitiva sostenible. Esta propuesta de valor, junto con la estrategia de marketing y el perfil competitivo adoptado por la empresa, constituirá su Matriz frente Externo - Frente Interno de la Imagen Competitiva y permitirá generar la lealtad de su mercado meta. Este recurso es quizás es el que más difícil se suele encontrar una PyME y en un contexto de crisis se siente su carencia.

Por último, el recurso que cita Amigo, (2018) como la capacidad empresarial, es quizás, uno de los más controvertidos, teniendo en cuenta la omnipresencia del empresario PyME, que muchas veces, así como es muy flexible, muchas decisiones las toma en soledad y sin suficiente análisis y planificación. El estilo de liderazgo que ejerce en un escenario de crisis puede ser audaz, dada la flexibilidad que le permite el tamaño y la estructura de la empresa.

¿Qué ventajas observamos puntualmente en las PyMEs respecto de las grandes empresas?:

- Flexibilidad: comparadas con las grandes empresas, las PyMEs pueden adaptarse con mayor facilidad a los cambios en el mercado, además pueden ajustar sus niveles de producción mucho más rápido que estas ante tales variaciones. 
- Cercanía: se relacionan mucho más con sus clientes, siendo esta relación más estrecha de manera que les permite conocer los deseos, gustos y necesidades, por lo que también pueden ofrecerles un trato más personalizado.

- Sencillez: tanto en su infraestructura como en su estructura organizativa, también permite ajustarse a los cambios rápidamente, incluso el hecho de cambiar de actividad, nicho de mercado y hasta la toma de decisiones son más expeditas (aunque se centralicen en el dueño o los socios de la PyME).

- Agilidad: no presentan tanta burocracia como las grandes empresas, el proceso de decisión es rápido

- Ocupación: aprovechan los nichos de mercado desocupados, los que no son tan interesante para las grandes empresas por resultarles poco rentables.

- Enfocadas: en la satisfacción de su cartera de clientes, están orientadas a ello.

- Versatilidad: tienen la capacidad de identificar nuevas oportunidades y abrirse campo en ellas para aprovechar nuevas oportunidades de negocio.

Fernández (2008) expresa que, al igual que sucedía con la eliminación de recursos obsoletos o no valiosos, las PyMEs no están sujetas a inercias organizativas y resistencia al cambio en el mismo grado que las grandes empresas, lo que puede favorecer su adaptación a entornos cambiantes.

Podemos sintetizar las principales desventajas en la gestión de las PyMEs, respecto de las grandes empresas:

$\checkmark$ Su administración no es especializada (lo empírico prevalece) y generalmente la realizan los propios dueños.

$\checkmark$ Por la propia inexperiencia administrativa del dueño, éste dedica un número mayor de horas al trabajo, con bajos rendimientos.

$\checkmark$ Mantienen altos costos de operación.

$\checkmark$ Escasa reinversión de utilidades para mejorar el equipo y las técnicas de producción.

$\checkmark$ El porcentaje de contratación de personal especializado y capacitado, es bajo por no poder abonar altos salarios.

$\checkmark$ Lo anterior provoca problemas de retención del personal especializado y con determinado expertise, puesto que los salarios y retribuciones que las PyMes proponen son inferiores a los de las grandes empresas.

$\checkmark$ La calidad de la producción no siempre es la mejor, existen ineficiencias porque los controles de calidad son mínimos o no existen. 
$\checkmark$ Atención oportuna de quejas y reclamos de clientes (aunque es un aspecto que evoluciona para mejor)

$\checkmark$ Menor control organizativo formalizado en comparación con las grandes empresas.

$\checkmark$ Tienen menor capacidad competitiva, tecnológica, publicitaria, económica y acceso a la financiación de la que tienen las grandes empresas.

$\checkmark$ Su inversión en I+D, equipos de trabajo y talento humano es escasa respecto a las grandes empresas.

$\checkmark$ Son organizaciones débiles en cuanto a la estandarización de productos.

$\checkmark$ Les resulta difícil competir con los precios que ofrecen las grandes empresas.

$\checkmark$ Sus posibilidades de fusión y absorción de empresas son reducidas o nulas.

En este sentido, Fernández (2008) expresa que “...las PyMEs no pueden obtener ventajas derivadas de la obtención de economías de escala y alcance, sus estrategias parecen ser fácilmente copiables, trabajan en nichos de mercado reducidos, tienen problemas para acceder a alta tecnología y disponen de recursos financieros limitados y basados principalmente en la generación de recursos internos".

\section{CAMBIOS CONDICIONANTES A PARTIR DEL COVID 19}

Ahora, qué fue lo que cambió en este contexto condicionado por la pandemia de COVID19?

Podemos decir que, dada la pandemia y la larga cuarentena, el Mercado cambió rápidamente. Desde el lado de las empresas, se aceleraron procesos que se venían gestando, como el cambio del modelo de negocio hacia la virtualidad (comercio electrónico, ferias virtuales y delivery), y desde el lado de la demanda, hacia una mayor revaloración del tiempo en casa, y dado la falta de salidas y de reuniones presenciales, una disminución de los consumos que hacen a la vanidad (ropa, perfumería, cosmética, zapatos, entre otros rubros). Estos cambios exigen una reacción urgente por parte de las empresas y es allí donde las PyMes tienen una oportunidad, teniendo en cuenta la rapidez para la toma de decisiones que se da en su interior, ya que son pocos quienes deciden (en PyMes básicamente los familiares). Esa celeridad se convierte en una ventaja respecto de las grandes empresas. Podemos decir que las PyMEs se adaptan y aprovechan mejor las nuevas oportunidades que ofrece este nuevo modelo de negocio.

También se ve favorecida la imagen de la PyMe y su relación con los clientes a partir de la flexibilidad, adaptabilidad y versatilidad, aprovechando la aceleración del proceso 
de digitalización así como la reestructuración de su negocio, modernizando su oferta de valor, una oportunidad de reinversión del negocio.

\section{LAS VENTAJAS Y DESVENTAJAS DE LAS GRANDES ORGANIZACIONES PARA GESTIONAR RECURSOS}

En cuanto a las grandes empresas, podemos focalizar la relevancia que le dan al proceso de planificación así como a los aspectos tecnológicos y a la introducción de nuevas tecnologías ( $1+D$ entre otras), el aprovechamiento de las sinergias mediante alianzas, acuerdos empresariales y/o entre empresas del grupo económico. Mediante estos últimos mecanismos la empresa se sirve de los recursos y capacidades de otras organizaciones para apalancar los suyos propios. Todo esto se traduce en eficiencia en el uso y gestión de los recursos

Siguiendo a Fernández y Suarez (1996) sobre la competencia como "lo que se hace" acercando el concepto de recurso al de capacidad, es decir un equipo de recursos para realizar alguna tarea o actividad, aquello que se hace bien como resultado del trabajo conjunto de una serie de recursos (Grant, 1991); es decir, lo que Hamel y Prahalad (1990) denominan competencia básica, si bien éstos se centran en los aspectos tecnológicos del saber hacer.

En las grandes empresas, aparece esa relación eficiente de recursos y capacidad reflejada en la planificación, en la aplicación de procesos de innovación, la generación de proyectos innovadores y nuevas tecnologías, que además favorecen la economía de escala.

Por otro lado, si aplicamos el modelo de recursos de Amigo (2009), descripto en el Punto II, al análisis de las ventajas y desventajas de las empresas grandes podemos destacar la eficiencia en la gestión de los recursos financieros, los recursos tecnológicos y la imagen corporativa.

En cuanto a los recursos financieros la gran empresa dado su tamaño puede disponer de un fuerte aporte por parte de las entidades financieras $y$, si se trata de una empresa cotizante, del mercado de capitales, local e internacional.

Donde las empresas grandes pueden tener una fortaleza es en la imagen corporativa, la propuesta de valor que la empresa haya logrado ser percibida por sus clientes, en relación a las acciones de sus competidores. Expresa Amigo (2009) que:

- "esa propuesta de valor debe ser ética, equitativa y no puede encapsularse en compartimentos estancos,

- la propuesta de marketing debe ser diferente y única para esta categoría de clientes, perceptible por el mercado y continua". 
La imagen corporativa resulta como consecuencia de un proceso continuo de comunicación y percepción de valor por parte de los clientes que se traducen en la lealtad hacia las diferentes propuestas de la empresa. $Y$ dicho proceso, podemos decir que resulta de la trayectoria que tiene la gran empresa apoyada por los recursos financieros.

Los recursos físicos quizás son un vagón en contra por el peso que significan los costos fijos, por lo que operan como una desventaja en situaciones de crisis.

En cuanto a los recursos humanos, la actividad de la empresa condicionará la mayor o menor flexibilidad para enfrentar la situación crítica. Teniendo en cuenta que en muchas organizaciones las tareas tienen un alto grado de especialización, y que un escenario de crisis exige versatilidad, el tiempo que requiere la recapacitación de los empleados operará como una desventaja en relación a empresas más pequeñas.

La misma cuestión que planteamos para las PyMES podemos platearlas para las grandes empresas y como se adaptaron y enfrentaron los cambios que operaron en el Mercado bajo la pandemia del COVID19

Los impactos que generan los cambios en el Mercado no tienen la misma respuesta en la gran empresa, dado que los procesos de toma de decisiones llevan más tiempo, teniendo en cuenta los procedimientos y las formalidades que exigen sus modelos de gestión.

Por otro lado, algunas grandes empresas (especialmente las tecnológicas) pudieron aprovechar una oportunidad a través de la generación de alianzas con el gobierno, apoyándolo con herramientas tecnológicas como los sistemas de rastreo, testeos y control de los habitantes respecto del COVID19. La fuerte relación que las grandes empresas habían desarrollado con el gobierno significa una ventaja, que debería ser apalancada, haciendo que el valor que genera esta alianza no se encapsule en compartimentos estancos. (Amigo; 2009; 2012, 2017).

\section{CONCLUSIONES}

- Las estrategias y los recursos son transversales a las empresas y se retroalimentan. Ante escenarios de imprevisibilidad e incertidumbre, este proceso se ve deflaccionado y perjudicado pues las empresas gestionan mejor sus recursos cuando aumenta su volumen de negocios, dado que los procesos decisorios se planifican y estructuran.

- Las estrategias se gestan en el frente interno gracias a la gestión de recursos y se ponen a prueba en el frente externo, en el negocio, desarrollando sus ventajas competitivas sostenibles, el frente externo agregará valor y se retroalimentara con el frente interno en tanto y en cuanto la empatía de la empresa por sus clientes, 
casi sea una misión empresarial.

- Las estrategias se formulan y se implementan para mejorar los vínculos con los clientes, dada la situación de vulnerabilidad de los mercados, los vínculos con los clientes, se deberán reforzar teniendo en cuenta el equilibrio entre sus requerimientos, sus expectativas y la oferta de la empresa.

- Las empresas están más preocupadas por los recursos generadores de costos que los generadores de valor, sin embargo en los escenarios planteados por la pandemia decretada por la OMS y antes la imprevisibilidad del futuro de las empresas en una economía con indicadores negativos, sólo los recursos generadores de valor, serán los impulsores de la rentabilidad y del crecimiento de las empresas.

- Recomendaciones: Establecer, formular e implementar políticas claras en la gestión de recursos que consistan en:

- Identificar áreas claves de decisión. Estas áreas claves son aquellas, que son funcionales a las estrategias de crecimiento, independientemente de las estructuras organizativas y con ellas también.

- Identificar los requerimientos que estas áreas funcionales poseen respecto de: Recursos Humanos; Recursos Físicos; Recursos Tecnológicos; Recursos Financieros, Imagen Corporativa;

- Establecer políticas de apalancamiento mutuas complementarias y mutuas excluyentes, identificando niveles de paridad y adversidad que determinarán lo que será necesario, formular, controlar y desarrollar, por ejemplo, qué impacto tiene en la imagen corporativa, la baja del precio de un producto, en la etapa de crecimiento de la demanda.

\section{REFERENCIAS BIBLIOGRÁFICAS:}

Amigo, Adriana (2013): Bases internas de la competitividad de las PyMEs. Impacto del área comercial en dichas bases, en PyMEs de las ciudades de Rosario, Resistencia y Corrientes. Trabajo presentado en las Decimoctavas Jornadas de Investigación de la Facultad de Ciencias Económicas y Estadísticas de la UNR, Noviembre 2013.

Amigo, Adriana (2018): La gestión de los recursos en las pymes, fuente de su competitividad interna. Trabajo presentado en las Vigesimoterceras Jornadas de Investigación de la Facultad de Ciencias Económicas y Estadísticas de la UNR., Noviembre 2018

Amigo, Adriana (2009): Negocios con valor, Editorial Fundación Ross, $1^{\circ}$ Ed. Rosario. Argentina 
Amigo, Adriana (2012): Negocios con valor, Editorial Fundación Ross, $2^{\circ}$ Ed. Rosario. Argentina

Amigo, Adriana (2017): Negocios con valor, una metodología para generar ventajas competitivas sostenibles. Editorial Académica Española, Ed. Europea. Madrid. España.

Carrión Maroto, Juan. (2006): Estrategia. De la Visión a la acción. ESIC. 2 • Edición. Madrid. España.

Fardelli Corropolese, Claudio; Díaz Daiana Valeria; González, Natalia Lorena; Szlechter, Diego Fabián (2012): Especificidades del proceso estratégico en PyMEs Argentinas: un Estudio de Casos, SaberEs Revista de Ciencias Económicas y Estadísticas, Número 4.

Fernández Rodríguez, Zulima y Suárez González, Isabel (1996): La Estrategia de la empresa desde una perspectiva basada en los recursos, Revista Europea de Dirección y Economía de la Empresa, Madrid.

Grant, Robert M. (2006): Dirección estratégica. Conceptos, técnicas y aplicaciones, Thomson Civitas, $5^{\mathrm{a}}$ edición, Madrid.

Hamel, Gary; Prahalad, Coimbatore Krishnarao (1999): Compitiendo por el futuro, Editorial Ariel, Sociedad Económica, 2º reimpresión, Barcelona. España.

Munuera Alemán José Luis; Rodríguez Escudero, Ana Isabel (2007): Estrategias de Marketing. Un enfoque basado en el proceso de dirección. ESIC. España 